\title{
AN EXPANSION METHOD FOR SINGULAR PERTURBATION PROBLEMS
}

\author{
J. J. MAHONY \\ (received 20 February 1960, revised 23 November, 1960)
}

\section{Summary}

A method is proposed for obtaining a uniformly valid perturbation expansion of the solution of a non-linear partial differential equation, involving either a large or small parameter, when the solution exhibits boundary layer type dependence on the parameter. The method differs from those previously in use in that it is not based on drawing a distinction between points in the boundary layer and points in the remainder of the field. Each point is treated as belonging to both regimes and this enables a stricter control to be maintained on the error terms in the expansions. The method is devised so as to ensure that all forms of error terms are reduced in order at each step in the expansion and not merely those error terms which are mathematically most significant for limiting values of the parameter. The perturbation series can then be used for a wider range of the parameter and provides a solution even when the boundary layer is not particularly thin.

The method is presented through its application to a problem which arises in the theory of the large deflexion of thin elastic plates but the principles underlying the method are more widely applicable.

\section{Introduction}

The concept of a boundary layer finds many applications in a wide field of applied mathematics problems but its usefulness has been restricted mainly to providing a first approximation to the solution. Recently several authors, Kaplun and Lagerstrom [1, 2, 3], Proudman and Pearson [4], have given a systematic method for obtaining higher order approximations to the solution of non-linear partial differential equations when the solution exhibits a boundary layer type dependence on a large or small parameter. In this method the solution is obtained in terms of two perturbation series, one valid in the boundary layer and the other valid in the remainder of the field. The terms in each series are rendered determinate by matching certain significant terms in each series, as decided by limiting processes. In this way 
the authors cited above were able to obtain the first few terms of the series for the particular problem they considered. It is a feature of the method that the two series have a common region of applicability and that in this region they are not identical when curtailed. In the case considered the difference is found to be small and reducible at each step of the expansion, as far as it has been carried, but it is a matter of speculation whether in general this will be true.

The method represents a marked advance as it permits the extension of the range of values of the parameter over which the perturbation method of solution can be used. In certain cases however the amount of extension is small due to the manner in which the two series are matched. As the result of the limiting processes, here typified by the small parameter $\varepsilon$ tending to zero, a term of the form $\exp (-k / \varepsilon)$, where $k$ is any positive number, is judged to be insignificant in comparison with any term of the form $\varepsilon^{n}$. Numerically this may not be so, especially if $k$ is somewhat small, for values of $\varepsilon$ for which it is desired to apply the perturbation expansion. The aim of this paper is to seek a method whereby such exponentially small, or other terms insignificant if the limit process is carried out, could be obtained. Such a method cannot rely on the limit process, $\varepsilon$ tending to zero, to decide the relative significance of different functional forms. But such limiting processes are essential for distinguishing the regions of validity of the two perturbation series. Thus in order to obtain the terms which are insignificant if the limit process is carried out, it is necessary to seek a single expansion valid over the entire field. An alternative criterion to the limit processes is needed to judge the progress of such a single expansion. The criterion which is used is that at the end of each step in the expansion the largest term, of each functional form of error term remaining in the differential equations and boundary conditions, shall be of smaller order than the term of corresponding form before the step.

The question of obtaining such a single expansion is examined for a particular problem, that of the solution of the Von Karman equations for the large deflexion of a thin cantilevered plate. This problem is chosen in preference to the particular problem considered by the authors cited above because their problem is special in that one of the series expansions is uniformly valid. For the problem chosen here a suitable procedure is developed for obtaining the required expansion and the solution process could be carried through although the arithmetic difficulties are considerable. As a practical method of solving the Von Karman equations the perturbation series, with exponentially small terms included, is not particularly promising though possibly no worse than the other methods available.

It is not suggested that the present procedure will apply without any adaptation to all singular perturbation problems. However the author 
believes that the ideas underlying the development of the procedure are applicable to a wide range of such problems. Thus the presentation follows the manner in which the procedure was obtained, namely as a series of modifications of the solution obtained using the method of the cited authors. In any further applications of the present method, much of the present work might be short-circuited but the insight gained from the present method of development may be valuable in any adaptations of the procedure. Perhaps of greatest interest are certain features of the solution for the particular problem, discussed in section 6 , which suggest that the method may be useful in dealing with other matters than extending the useful range of the perturbation series which was the original aim.

\section{Background to the problem}

As interest here is centered on the method of obtaining solutions, rather than on the details of any particular physical problem, a semi-inverse method is adopted to reduce the amount of algebra. Thus from a physical problem, treated by Mahony $[5,6]$, the following reduced mathematical problem is formulated. Given an arbitrary integrable function $p(x, y)$, find an asymptotic expansion of the solution of the differential equations

$$
\begin{gathered}
\nabla^{\mathbf{4}} F=\omega_{x y}^{2}-\omega_{x x} \omega_{y y} \\
\nabla^{\mathbf{4}} \omega=L p(x, y)+F_{x x} \omega_{y y}+F_{y y} \omega_{x x}-2 F_{x y} \omega_{x y}
\end{gathered}
$$

which satisfies the boundary conditions,

$$
\begin{gathered}
\omega_{y y}(x, 0)+\mu \omega_{x x}(x, 0)=0 \\
\omega_{y y y}(x, 0)+(2-\mu) \omega_{x x y}(x, 0)=0 \\
F(x, 0)=F_{y}(x, 0)=0
\end{gathered}
$$

and

$$
\begin{aligned}
& \omega_{y}(x, 1)=\omega_{y y y}(x, 1)=0 \\
& F_{y}(x, 1)=F_{y y y}(x, 1)=0
\end{aligned}
$$

where $L$ is a large parameter and $\mu$ is a constant neither large nor small. Mahony $[5,6]$ has obtained an asymptotic expansion of the solution for the case when exponentially small terms may be neglected. The method used was essentially that of Kaplun, Lagerstrom, Proudman and Pearson $[1,2,3,4]$ modified to yield a single expansion and the results wanted for the present work will be reviewed briefly.

Two series representations of function $\omega(x, y, L)$ can be obtained using limit processes attributed to Kaplun [9] by Lagerstrom and Cole [7] who 
give a more complete exposition. If $(x, y)$ are held fixed with $y \neq 0$, the set of functions $W_{n}(x, y)$ may be defined by the limit processes

and

$$
\operatorname{limit}_{L \rightarrow \infty} L^{-1} \omega(x, y, L)=W_{0}(x, y)
$$

$$
\operatorname{limit}_{L \rightarrow \infty} L^{-1+\frac{1}{2} n}\left\{\omega(x, y, L)-\sum_{r=0}^{n-1} L^{1-\frac{1}{2} r} W_{r}(x, y)\right\}=W_{n}(x, y)
$$

while if $(x, \eta)$ are held fixed, where

$$
\eta=L^{\frac{1}{2}} y,
$$

another set of functions $\mathscr{W}_{n}(x, \eta)$ is defined by

$$
\operatorname{limit}_{L \rightarrow \infty} \omega(x, y, L)=\mathscr{W}_{0}(x, \eta)
$$

and

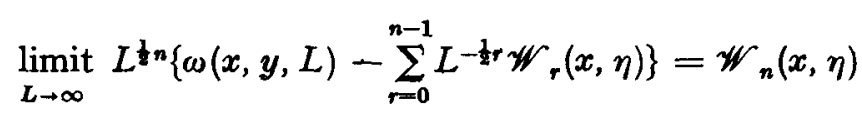

It follows that the asymptotic series

$$
W(x, y, L)=L W_{0}(x)+L^{\frac{1}{2}} W_{1}(x)+\sum_{n=2}^{\infty} L^{1-\frac{1}{2} n} W_{n}(x, y)^{*}
$$

and

$$
\mathscr{W}(x, \eta, L)=\sum_{n=0}^{\infty} L^{-\frac{1}{n} n} \mathscr{W}_{n}(x, \eta)
$$

provide approximations, in restricted regions, to the solution $\omega(x, y, L)$. The function $\mathscr{W}$ is interpreted as the approximation to the solution in a boundary layer of thickness $O\left(L^{-\mathbf{t}}\right)$ along the edge $y=0$ while $W$ is interpreted as the approximation to the solution in the remainder of the field.

Certain types of terms are selected in both types of limit processes. Thus if the function $W_{n}(x, y)$ is expanded in a power series in $y$ and this series is rearranged in terms of $\eta$ it can be seen that the resultant terms,

$$
\sum_{m=0}^{\infty} W_{n}^{(m)}(x, 0)(m !)^{-1} L^{-1 m} \eta^{m}=W_{n}(x, y)
$$

must occur in the expansion of $\mathscr{W}(x, \eta, L)$. It is just such terms which are matched when the technique of Kaplun, Lagerstrom, Proudman and Pearson is applied to the present problem. If all terms common to both $W$ and $\mathscr{W}$ are removed from $\mathscr{W}$ leaving $\mathscr{W}^{*}$ it is a consequence of the matching procedure that $\mathscr{W}^{*}$ is negligible in comparison with any power of $L^{-\frac{1}{2}}$ in the limit $L$

* The functions $W_{0}$ and $W_{1}$ are found to be independent of $y$. 
tending to infinity for $y$ fixed and non-zero. Thus the combination $(W, \mathscr{W})$ provides a single asymptotic expansion of $\omega$ everywhere for, inside the boundary layer, it is equal to $\mathscr{W}$ while, outside the boundary layer, it differs from $W$ inessentially. In cases more general than the present problem it may be necessary to consider such cases as, for example, $W_{n}$ expansible as a Laurent rather than as a power series. In such a case, a reduced function $W^{*}$ is obtained from $W$ by extracting all negative powers of $y$ which can be seen to occur also as negative powers of $\eta$. In such a case the single approximation to the solution $\omega$ is obtained as $\left(W^{*}+\mathscr{W}^{*}\right)$. It can be seen that the arrangement of the common terms as either functions of $y$ in $W^{*}$ or functions of $\eta$ in $\mathscr{W}^{*}$ is made so that each common term appears in that form which indicates the biggest order anywhere in the field. For example, a term $L^{-(m+n) / 2} \eta^{m}$, which can be rearranged in any of the equivalent forms $L^{-(r+n) / 2} \eta^{r} y^{m-r}$, is to appear as $L^{-\frac{1}{n} n} y^{m}$ for the largest order of this term is that of $L^{-\frac{1}{2} n}$ occurring when $y$ is $O(1)$ and consequently $\eta$ is $O\left(L^{\frac{1}{2}}\right)$. The motivation behind this arrangement is to ensure that no subsequent term in the series will be of as large an order in $L$ as any previous term. Such would not be the case for example if $\omega_{1}$ behaved like a $\eta$ for large $\eta$ for then $L^{-\frac{1}{2}} \omega_{1}$ would be equal to $a y$ and hence of unit order over most of the field. When successive terms of the series are of descending order the series will be usefully asymptotic.

As a consequence of the above methods of construction it follows that the solution is expansible in the form

$$
\omega=L W_{0}(x)+L^{\frac{1}{2}} W_{1}(x)+\sum_{n=0}^{\infty} L^{-\frac{1}{2} n}\left\{W_{n+2}(x, y)+\omega_{n}(x, \eta)\right\}
$$

where the star notation has been dropped. Further

$$
\begin{gathered}
\operatorname{limit}_{\eta \rightarrow \infty} \omega_{n}(x, \eta)=0 \\
\underset{y \rightarrow 0}{\operatorname{limit}} W_{n}(x, y) \text { is finite }
\end{gathered}
$$

since all singular terms have been removed from the functions undergoing the limit process.* Similarly the function $F$ is expansible in the form

$$
F=\sum_{n=0}^{\infty} L^{-\ln n}\left\{F_{n}(x, y)+f_{n}(x, \eta)\right\}
$$

where the $F_{n}$ and $f_{n}$ have similar properties to $W_{n}$ and $\omega_{n}$. The error terms in these expansions remain to be assessed. The functions $W_{n}$ and $\omega_{n}$ can be determined successively by inserting the expansions (5) in the differential equations (1), the boundary conditions (2), equating the coefficients of the

- Here it will be assumed that $\omega$ cannot be exponentially large as $\eta \rightarrow \infty$ for physical reasons. This point will be examined further in section 4 . 
various powers of $L^{-\frac{1}{2}}$ and using equations (6) and their counterparts for $F_{n}$ and $f_{n}$. These are found to be sufficient to determine the various terms uniquely. One feature of the solution procedure is that having assumed the form of expansion (5) and the properties (6) there is no difficulty encountered if, as can happen in properly set physical problems, $F(\dot{x}, y)$ is not expansible in series form about $y=0$. Thus it appears that, although the present method has been derived from the work of Kaplun, Lagerstrom, Proudman and Pearson, it is applicable in cases where the other method must fail.

Mahony [6] gives solutions for $W_{0}(x)$ and $W_{1}(x)$ in terms of the function $p(x, y)$ and henceforth these will be treated as known functions although their specific form is of little importance here. He also gives solutions for $\omega_{0}$ and $\omega_{1}$ and in the slightly changed notation of the present paper they are

$$
\omega_{0}=-\mu \operatorname{sign} W_{0}^{\prime \prime}(x)\{\cos k \eta-\sin k \eta\} \exp (-k \eta)
$$

where $k$ is the real positive root of

and

$$
4 k^{4}=\left\{W_{0}^{\prime \prime}(x)\right\}^{2}
$$

$$
\begin{gathered}
\omega_{1}=\mu W_{1}^{\prime \prime}(x) \exp (-k \eta)\left\{k \eta(\cos k \eta-\sin k \eta)+\left(1+k^{-1}\right) \cos k \eta\right. \\
\left.-\left(1-2 k^{-1}\right) \sin k \eta\right\} .
\end{gathered}
$$

An examination of these two terms reveals that this solution is unsatisfactory if exponentially small terms are not negligible. This is because, for large values of $\eta$, it can be shown that

$$
\begin{aligned}
\omega_{0}-L^{-\frac{1}{2}} \omega_{1} & =\omega_{0}\left\{1-k W_{1}^{\prime \prime} \operatorname{sign} W_{0}^{\prime \prime} L^{-\frac{1}{2}} \eta\right\} \\
& \approx \omega_{0}\left\{1-k W_{1}^{\prime \prime} \operatorname{sign} W_{0}^{\prime \prime} y\right\}
\end{aligned}
$$

when the positive power of $\eta$ is arranged as a term in $y$. It follows that $L^{-\frac{1}{2}} \omega_{1}$ is as large as $\omega_{0}$ over most of the field where they are both $O\left[\exp \left(-L^{\frac{1}{2}}\right)\right]$.* $^{*}$ Thus $\omega_{0}$ does not provide a valid approximation to the portion of the solution which is of this order. Moreover neither does $\omega_{0}+L^{-\frac{1}{2}} \omega_{1}$ since, as typically happens in singular perturbation problems of non-linear differential equations, portions of the subsequent terms $L^{-\frac{1}{2} n} \omega_{n}$ will be of the same order. This is easy to see in the present problem for the functions $\omega_{n}$ are determined from differential equations of the pattern

$$
\omega_{n \eta \eta \eta \eta}+4 k^{4} \omega_{n}=a_{n}(x) \omega_{n-1}+\mathscr{R}_{n}
$$

* For convenience this notation will be used, where there is no ambiguity, to denote any term $K$ such that

$$
\operatorname{limit}_{L \rightarrow \infty} \mathrm{K} \exp (k \eta) /\{\cos k \eta-\sin k \eta\}
$$

is bounded, non-zero for fixed values of $x$ and $y \neq 0$. 
Because of the "resonance" between $\omega_{n}$ and $\omega_{n-1}$ there will be a cumulative growth of the functions $\omega_{n}$ for large $\eta$ of the form

$$
\omega_{n} \approx b_{n}(x) \eta^{n} \omega_{0} \text {. }
$$

Hence terms $O\left[\exp \left(-L^{\frac{1}{2}}\right)\right]$ will not be obtained correctly if the series (5) are curtailed after any finite number of terms.

Since only a finite number of terms can be obtained in practice it is necessary to find some means of summing the contributing portion of all the $\omega_{n}$. A formal summation may be achieved by rearranging the positive powers of $\eta$ in terms of $y$ and the result obtained

$$
\omega_{0} \sum_{n=0}^{\infty} L^{-\frac{1}{2} n} b_{n} \eta^{n}=w_{0} \sum_{n=0}^{\infty} b_{n}(x) y^{n}
$$

indicates that the term $O\left[\exp \left(-L^{\frac{1}{2}}\right)\right]$ is of the form $\omega_{0}(x, \eta) g_{0}(x, y)$ or more briefly $\omega_{0}(x, y, \eta)$. If this summation has been achieved successfully there can be no resonant forcing term in the differential equation for $\omega_{1}$. Thus the next term in the expansion will be $O\left[L^{-\frac{1}{2}} \exp \left(-L^{\frac{1}{2}}\right)\right]$ and this will be of uniformly smaller order than $\omega_{0}$. This summation process is seen to imply that positive powers of $\eta$ should be re-arranged even when, multiplying functions such as exponentials, the composite term satisfies equation (6a). Since the function $\omega_{1}$ will now be of the same form as the original $\omega_{0}(x, \eta)$ a repetition of the previous argument implies that $\omega_{1}$ must be written in the form $\omega_{1}(x, y, \eta)$ and further repetition shows that all the $\omega_{n}$ are of this same form. All these $\omega_{n}$ are to be such that they have no positive powers of $\eta$ involved in their expansions for large $\eta$ nor negative powers of $y$ involved in its expansion for small $y$.

\section{Trial expansion}

The above arguments suggest trying the formal expansions

$$
\begin{gathered}
\omega=L W_{0}(x)+L^{\frac{1}{2}} W_{1}(x)+\sum_{n=0}^{\infty} L^{-\frac{1}{2} n}\left\{W_{n+2}(x, y)+\omega_{n}(x, y, \eta)\right\} \\
F=\sum_{n=0}^{\infty} L^{-\frac{1}{2}}\left\{F_{n}(x, y)+f_{n}(x, y, \eta)\right\}
\end{gathered}
$$

and if these are substituted into equations (1) and the coefficients of the three highest powers of $L$ are equated to zero the equations obtained are

$$
\begin{gathered}
f_{0 \eta \eta \eta}+W_{0}^{\prime \prime}(x) \omega_{0 \eta \eta}=0 \\
\omega_{0 \eta \eta \eta \eta}-W_{0}^{\prime \prime}(x) f_{0 \eta \eta}=0 \\
f_{1 \eta \eta \eta \eta}+W_{0}^{\prime \prime}(x) \omega_{1 \eta \eta}=-4 f_{0 \eta \eta \eta \nu}-2 W_{0}^{\prime \prime} \omega_{0 \eta y}-W_{1}^{\prime \prime} \omega_{0 \eta \eta} \\
\omega_{1 \eta \eta \eta \eta}-W_{0}^{\prime \prime} f_{1 \eta \eta}=-4 \omega_{0 \eta \eta \eta \nu}+2 W_{0}^{\prime \prime} f_{0 \eta y}+W_{1}^{\prime \prime} f_{0 \eta \eta}
\end{gathered}
$$




$$
\begin{aligned}
f_{2 \eta \eta \eta \eta}+W_{0}^{\prime \prime}(x) \omega_{2 \eta \eta}= & -4 f_{1 \eta \eta \eta v}-6 f_{0 \eta \eta y y}-2 f_{0 x x \eta \eta}+\omega_{0 x \eta}^{2} \\
& -\omega_{0 x x} \omega_{0 \eta \eta}-W_{0}^{\prime \prime}\left(\omega_{0 y y}+2 \omega_{1 \eta \eta}\right) \\
& -W_{1}^{\prime \prime}\left(\omega_{1 \eta \eta}+2 \omega_{0 \eta y}\right) \\
\omega_{2 \eta \eta \eta \eta}-W_{0}^{\prime \prime}(x) f_{2 \eta \eta}= & p(x, y)-W_{0}^{\mathrm{IV}}(x)+W_{0}^{\prime \prime}(x)\left(f_{0 y y}+F_{0 y y}\right) \\
& -4 \omega_{1 \eta \eta \eta \nu}-6 \omega_{0 \eta \eta v y}-2 \omega_{0 x x \eta \eta}-2 f_{0 x \eta} \omega_{0 x \eta} \\
& +W_{0}^{\prime \prime}\left(f_{2 \eta \eta}+2 f_{1 \eta \nu}\right)+W_{1}^{\prime \prime}\left(f_{1 \eta \eta}+2 f_{0 \eta \nu}\right) \\
& +W_{2}^{\prime \prime} f_{0 \eta \eta} .
\end{aligned}
$$

If equations $(8 \mathrm{a}, \mathrm{b})$ are integrated twice with respect to $\eta$ and allowance is made for the fact that $\omega_{0}$ and $f_{0}$ can contain no positive powers of $\eta$ then the equations reduce to

$$
\begin{aligned}
& f_{0 \eta \eta}+W_{0}^{\prime \prime}(x) \omega_{0}=0 \\
& \omega_{0 \eta \eta}-W_{0}^{\prime \prime}(x) f_{0}=0 .
\end{aligned}
$$

The solution for $\omega_{0}$ which is not exponentially large for large values of $\eta$ is

$$
\omega_{0}(x, y, \eta)=\exp (-k \eta)\{A(x, y) \cos k \eta+B(x, y) \sin k \eta\}
$$

where $A$ and $B$ are arbitrary functions. The boundary conditions on $y=\eta=0$, satisfied by $\omega_{0}$, derive from the terms $O(L)$ and $O\left(L^{\frac{3}{2}}\right)$ when the expansions (7) are substituted into equations (2). These boundary conditions will be satisfied provided

$$
\begin{gathered}
A(x, 0)=-\mu \operatorname{sign} W_{0}^{\prime \prime}(x) \\
B(x, 0)=\mu \operatorname{sign} W_{0}^{\prime \prime}(x)
\end{gathered}
$$

and the solution for $A$ and $B$ cannot be completed from the leading order terms alone. However if a similar procedure is applied to equations (9) and use is made of the known properties of $\omega_{0}(x, y, \eta)$ it can be shown that

$$
f_{1 \eta \eta}+W_{0}^{\prime \prime} \omega_{1}=-W_{1}^{\prime \prime} \omega_{0}+2 W_{0}^{\prime \prime} \frac{\partial}{\partial y} \int_{\infty}^{\eta} \omega_{0} d \eta
$$

and

$$
\omega_{1 \eta \eta}-W_{0}^{\prime \prime} f_{1}=-W_{0}^{\prime \prime} W_{1}^{\prime \prime} \omega_{0}-2 W_{0}^{\prime \prime} \omega_{0 \eta y} .
$$

The right hand sides of these equations contain resonant forcing terms for general functions $A$ and $B$, but it has been seen already that such terms would lead to an expression for $\omega_{1}$, which contains terms such that $L^{-\frac{1}{2}} \omega$, is as large as $\omega_{0}$ for large values of $\eta$. In order to prevent this, and so obtain a suitably asymptotic series, it is necessary to dispose $A$ and $B$ such that the resonant forcing terms are absent. It can be shown that this is possible if $A$ and $B$ satisfy the differential equations 
where

$$
\begin{aligned}
& B_{y}+A_{y}+2 \sigma A=0 \\
& B_{y}-A_{y}+2 \sigma B=0
\end{aligned}
$$

$$
\sigma=W_{1}^{\prime \prime}(x) /\left[4 k(x) W_{0}^{\prime \prime}(x)\right]
$$

and then the equations for $\omega_{1}$ and $f_{1}$ reduce to

$$
\begin{aligned}
& f_{1 \eta \eta}+W_{0}^{\prime \prime} \omega_{1}=0 \\
& \omega_{1 \eta \eta}-W_{0}^{\prime \prime} f_{1}=0 .
\end{aligned}
$$

The values of $A(x, 0), B(x, 0)$ have been obtained previously so that the required solutions for $A$ and $B$ may be shown to be

and

$$
A(x, y)=-\mu \operatorname{sign} W_{0}^{\prime \prime}(x) \exp (-\sigma y)\{\cos \sigma y-\sin \sigma y\}
$$

$$
B(x, y)=\mu \operatorname{sign} W_{0}^{\prime \prime}(x) \exp (\sigma y)\{\cos \sigma y+\sin \sigma y\}
$$

and so the solution for the leading order exponential term has been obtained. The solution for $\omega_{1}$, which is not exponentially large for large $\eta$, can be shown to be

$$
\omega_{1}(x, y, \eta)=\exp (-k \eta)\{C(x, y) \cos k \eta+D(x, y) \sin k \eta\}
$$

where $C$ and $D$ are arbitrary functions whose values on $y=0$ can be found from the boundary conditions (2).

It is possible to achieve an equivalent approximation to the term $\omega_{0}$ by an entirely separate method which derives from Poincarés method for obtaining the perturbed frequency of a non-linear oscillatory system. In this method, instead of introducing the functions $A(x, y), B(x, y)$ as above, the variable $k$ in the exponential terms is also expanded in powers of $L^{-\frac{1}{2}}$ and the higher terms in the expansion for $k$ are chosen so that the resonant forcing terms do not appear in the equation for $\omega_{1}$. Actually the two methods yield only a rearrangement of the same series in the present case. In the treatment of non-linear oscillatory problems interest centres on the perturbed frequency, essentially $k$, so that Poincarés treatment is more suitable. In the present context where interest centres on $\omega$, it is convenient if resonance in the equation for $\omega_{2}$ or any higher term does not influence $\omega_{0}$, once it has been obtained, so that the present method has a slight advantage. Because of the close relationship between Poincare's method and the work of Lighthill [8] it would appear that the method outlined here may provide an alternative method to the use of Lighthill's method in many problems.

However when a similar procedure is used in an attempt to determine the functions $C(x, y)$ and $D(x, y)$ in the term $\omega_{1}$ a serious difficulty is encountered. Before equations (10) can be used for the determination of $\omega_{2}$ it is 
necessary to apply the limit process with $L$ tending to infinity with $x$ and $y$ fixed so that all the terms involving derivatives with respect to $\eta$ vanish and there remains

$$
O=p(x, y)-W_{0}^{\mathrm{IV}}(x)+W_{0}^{\prime \prime}(x) F_{0 y}
$$

which Mahony [6] has used to determine $W_{0}$ and $F_{0}$. With these terms cancelled from equation (10b) only terms involving the functions $\omega_{n}$ and $f_{n}$ remain in equations (10). If these equations are now considered, the functional dependence on $\eta$ of the terms on the right hand sides of these equations is of one of the three forms

$$
\begin{gathered}
\exp (-k \eta)\{\cos k \eta, \sin k \eta\} \\
\eta^{2} \exp (-2 k \eta)\left\{\cos ^{2} k \eta, \sin ^{2} k \eta, \sin 2 k \eta\right\} \\
\eta^{2} \exp (-k \eta)\{\cos k \eta, \sin k \eta\} .
\end{gathered}
$$

Terms of the first type can be treated as before. The only point of interest in such terms is that the differential equations for $C$ and $D$ involve $A$ so there will be a resonance in this determination as well. This is of no significance in the present work where $y$ is restricted to a range of unit order. If the range of $y$ were infinite this point would need further investigation. The second form is not resonant so that for large $\eta$ it would contribute a term like

$$
\eta^{2} \exp (-2 k \eta)\left\{\cos ^{2} k \eta, \sin ^{2} k \eta, \sin 2 k \eta\right\}
$$

or

$$
L y^{2} \exp (-2 k \eta)\left\{\cos ^{2} k \eta, \sin ^{2} k \eta, \sin 2 k \eta\right\}
$$

to $\omega_{2}$ so that the rearranged form implies $L^{-1} \omega_{2}$ is formally of the same order as $\omega_{0}$. This type of term might not be significant because the exponential term is smaller because of the factor 2. However, if it is significant, no adjustment of $C$ and $D$ or application of Poincaré's or Lighthill's techniques will remove this difficulty. Neither will any of these methods cope with the even more serious third term which indicates the complete failure of the term $\omega_{0}(x, y, \eta)$ to approximate the exponentially small term. For, since the third form of term is resonant it leads to a term in $\omega_{2}$, for large $\eta$, of the form

$$
\eta^{3} \exp (-k \eta)\{\cos k \eta, \sin k \eta\}
$$

or

$$
L^{\frac{3}{2}} y^{3} \exp (-k \eta)\{\cos k \eta, \sin k \eta\}
$$

so that $L^{-1} \omega_{2}$ is of greater order than $\omega_{0}$ over the majority of the field. It might be thought that this indicates that $\omega$ should include a term $O\left[L^{\frac{1}{2}} \exp \right.$ $\left.\left(-L^{\frac{1}{2}}\right)\right]$ which would appear in the expansion of $\omega$ as $L^{\frac{1}{2}} \omega_{-1}$. If such a term is included it replaces $\omega_{0}$ as the leading order term and the difficulty moves 
forward to indicate the need for a term $L \omega_{-2}$ etc. This interpretation cannot supply the solution to the difficulty. The only alternative appears to be to arrange the troublesome term in the differential equation in the form $L y^{2} \exp (-k \eta)\{\cos k \eta, \sin k \eta\}$ and include this term back in the equation for determining $\omega_{0}$. It is not to be taken literally that this expression should be substituted in the equation for $\omega_{0}$ for this term derives from terms like $\omega_{0 x x \eta \eta}$ and the substitution invalidates the original solution. What is meant is that certain terms like $\omega_{0 x x \eta \eta}$ which have been estimated formally as $O(L)$ should be rearranged so that they are re-estimated as $O\left(L^{2}\right)$ and hence included in the equation for $\omega_{0}$.

Before this can be done it is necessary to discover what it is about the difficult terms like $\omega_{0 x x \eta \eta}$ which makes the rearrangement necessary. It is useful to examine the discredited solution $\omega_{0}(x, y, \eta)$ for this purpose. The presence of the positive powers of $\eta$ can be traced to the process of differentiation with respect to $x$. Thus

$$
\begin{aligned}
\frac{\partial}{\partial x}\{A(x, y) \exp (-k \eta) \cos k \eta\}=A_{x} & \exp (-k \eta) \cos k \eta \\
& -A k^{\prime}(x) \eta \exp (-k \eta)\{\cos k \eta+\sin k \eta\}
\end{aligned}
$$

and it is only the second term which is to be rearranged as formally $O\left(L^{\frac{1}{2}}\right)$. Thus certain derivatives taken with respect to $x$ result in an increase of the formal order of the term just as derivatives taken with respect to $y$ increase the order when they act through $\eta$. In the above function it is those derivatives which act through the combination $k(x) \eta$ which raise the order by a factor $L^{\frac{1}{2}}$. This suggests looking for a new variable

$$
\zeta=L^{\frac{1}{2}} u(x, y)
$$

where $u$ is at present arbitrary, which is to play the same role for a valid solution as the combination $k(x) \eta$ does for $\omega_{0}(x, y, \eta)$. The aim will be to dispose the function $u(x, y)$ so that when differentiating $\omega(x, y, \zeta, L)$ with respect to $x$ or $y$ only those derivatives involving $\omega_{\xi}$ are of order greater than $\omega$. The previous considerations suggest the need for at least one such variable and this is found to be all that is necessary for the present problem. In other problems more than one may be necessary but probably this will be indicated just as the need for at least one has been indicated in this case.

It is of interest to obtain an interpretation of the variable $\zeta$. In conventional boundary layer theory the layer is assumed so thin that all exponential terms are negligible other than for $y$ small. Thus in this case the variable $\eta$ which gives the direction of the greatest gradients can be introduced as the scaled normal distance from the boundary as in equation (4). The work of Kaplun [9] suggests that any co-ordinate system locally similar to $(x, \eta)$ 
at $y=0$, may be used to describe the boundary layer in the limit as $L$ tends to infinity, which corresponds to the case when the boundary layer can be regarded as of infinitesimal thickness. However when exponentially small terms are being sought, the boundary layer cannot be considered as confined to a vanishingly small neighbourhood of $y=0$ and thus a choice has to be made between the infinitely many co-ordinate systems possible in that case. Unlike in the work of Kaplun [9], this choice is not to be made so that the boundary layer solution gives a uniform approximation to the solution throughout the entire field in the limit as $L$ tends to infinity. Such an approximation has been obtained here already by using an expansion of the form (5). Here the variable $\zeta$, the boundary layer co-ordinate, is to be chosen to indicate at any point in the field, and not merely at the boundary, the direction of the greatest rate of change in the "boundary layer portion" of the solution.

The role, which the variable $\zeta$ is required to play, places severe restrictions on the form in which $\zeta$ can occur. A term of the form $\exp \{\alpha(x, y) \zeta\}$, for example, is possible only if $\alpha$ is a constant. For if $\alpha$ varied with $x$, the result of differentiation with respect to $x$ would be

$$
\exp \{\alpha(x, y) \zeta\}\left[\alpha(x, y) L^{\frac{1}{2}} u_{x}+L^{\frac{1}{2}} u \alpha_{x}\right]
$$

but the last term involves an increase in order without a differentiation with respect to $\zeta$, thus infringing the requirement by which it is hoped to define $\zeta$. Similarly $\alpha$ does not vary with $y$ so that $\alpha$ must be constant. The same result applies to trigonometric functions and this covers all the cases encountered in the present problem. In other cases it would appear that for any function of the form $\phi(\beta \eta)$, which led to difficulties of the type which here indicated the need for a variable $\zeta$, the argument could be applied to indicate that $\phi(\alpha \zeta)$ requires $\alpha$ constant. Further the requirement of successive diminution in the order of the terms of the expansion requires that no positive power of $\zeta$ should appear apart from as an argument in a suitable functional form. For example exp $\zeta$ is suitable, while $\zeta \exp \zeta$ and $\zeta$ are not as their order is more correctly indicated by $L^{\frac{1}{2}} u \exp \zeta$ and $L^{\frac{1}{2}} u$.

It has been noted previously that the expansion of the form given by equations (5) provides a satisfactory solution if exponentially small terms are neglected. It follows therefore that, under the strict mathematical limit process $x, L^{\frac{1}{2}} u$ fixed, $L$ tending to infinity, the solution expressed in terms of $(x, y, \zeta)$ must approach the earlier form of solution. Thus it follows that

$$
u(x, y)=\text { const. }\{k(x) y+o(y)\}
$$

for sufficiently small values of $y$. The value of this constant has no signifi- 
cant effect on the solution and the definition of $\zeta$ will be completed by requiring that it is unity.

\section{Solution}

The considerations of the previous section suggest that an asymptotic solution with the required properties may be obtained from series of the form

$$
\omega=L W_{0}(x)+L^{\frac{1}{2}} W_{1}(x)+\sum_{n=0}^{\infty} L^{-\frac{1}{2} n}\left\{W_{n+2}(x, y)+\omega_{n}(x, y, \zeta)\right\}
$$

and

$$
F=\sum_{n=0}^{\infty} L^{-\frac{1}{2} n}\left\{F_{n}(x, y)+f_{n}(x, y, \zeta)\right\}
$$

Thus it follows from equation (11) that

$$
\omega_{x}=L W_{0}^{1}+L^{\frac{1}{2}} W_{1}^{\prime}+\sum_{n=0}^{\infty} L^{-\frac{1}{2} n}\left\{W_{n+2_{x}}+\omega_{n_{x}}+L^{\frac{1}{2}} \omega_{n_{\zeta}} u_{x}\right\}
$$

and

$$
\begin{aligned}
\omega_{x x}=L W_{0}^{\prime \prime}+L^{\frac{1}{2}} W_{1}^{\prime \prime}+\sum_{n=0}^{\infty} L^{-\frac{1}{2} n}\left\{W_{n+2_{x x}}+\omega_{n_{x x}}\right. & +L^{\frac{1}{2}}\left(2 \omega_{n_{x \zeta}} u_{x}\right. \\
& \left.\left.+\omega_{n_{\zeta}} u_{x x}\right)+L \omega_{n_{\zeta \zeta}} u_{x}^{2}\right\}
\end{aligned}
$$

etc., where the derivatives of $\omega$ on the left hand sides are taken with $y$ constant while those of $\omega_{n}$ on the right hand side are taken with both $y$ and $\zeta$ constant. It is apparent that the largest order term in $\omega_{x x}$ is $\left\{L W_{0}^{\prime \prime}+L u_{x}^{2} \omega_{0 \nvdash \zeta}\right\}$ and this would suggest that the equation for $\omega_{0}(x, y, \zeta)$ will become non-linear and thus be greaty different to the one for $\omega_{0}(x, y, \eta)$. However when this point is examined more closely it is found that all the non-linear terms cancel from the equation for $\omega_{0}(x, y, \zeta)$ and it is found to be similar to the equation for $\omega_{0}(x, y, \eta)$. This cancellation of terms, the author feels, is not due to lucky chance but is due to the fact that the original boundary layer solution was correct in essence with errors only in the exponentially small terms. Thus it is not to be expected that the present solution form, which should be closely equivalent to solution form (5) if $u$ is replaced by $k(x) y$, will be of significantly different conformation.

If the expansions (12) are substituted in equations (1) then the terms which are formally $O\left(L^{2}\right)$ yield

and

$$
\left(u_{x}^{2}+u_{y}^{2}\right)^{2} f_{0_{5 \zeta \zeta}}=-\cdots W_{v}^{\prime \prime}(x) u_{y}^{2} \omega_{0 \zeta 5}
$$

$$
\left(u_{x}^{2}+u_{v}^{2}\right)^{2} \omega_{0_{5 \zeta \zeta 5}}=W_{0}^{\prime \prime}(x) u_{v}^{2} f_{0_{5 \zeta}}
$$


If the same procedure is followed as for the previous determination of $\omega_{0}(x, y, \eta)$ it can be shown that these equations have particular solutions of the form

$$
\exp \{( \pm 1 \pm i) \alpha(x, y) \zeta\}
$$

where $\alpha$ involves $u_{x}, u_{y}$ and $W_{0}^{\prime \prime}$. Such a solution is permissible only with $\alpha$ constant, if $\zeta$ is to have the required properties, and hence it can be shown that

$$
\left(u_{x}^{2}+u_{y}^{2}\right)^{2}=\text { const. } W_{0}^{\prime \prime}(x) u_{y}^{2}
$$

This constant can be evaluated by noting that for small $y$

$$
u=k(x) y+o(y)
$$

and that $k$ is related to $W_{0}$ through

$$
4 k^{4}=\left\{W_{0}^{\prime \prime}(x)\right\}^{2}
$$

so that the above equation reduces to

$$
u_{x}^{2}+u_{v}^{2}=k(x)\left|u_{y}\right| .
$$

The question of solving this equation for $u$ is examined in the next section and, for the remainder of this section, it will be assumed that a solution has been found in which $u$ is non-negative throughout the field.

With this choice of $u$, it is possible to reduce the above equations for $\omega_{0}$ and $f_{0}$ to the ordinary differential equation

$$
\omega_{0_{\zeta \zeta \zeta \zeta}}=-4 \omega_{0}
$$

which has the general solution

$$
\begin{aligned}
\omega_{0}=\{A(x, y) \cos \zeta & +B(x, y) \sin \zeta\} \exp (-\zeta) \\
& +\{C(x, y) \cos \zeta+D(x, y) \sin \zeta\} \exp \zeta
\end{aligned}
$$

If the previous treatment were followed $C$ and $D$ would be omitted as making $\omega_{0}$ exponentially large but then the boundary conditions (3) could not be satisfied. Throughout the previous analysis an inverse approach has been adopted with regard to these particular boundary conditions and exponentially small terms accepted. Here it may be shown that this is not necessary. The boundary conditions (3) can be satisfied exactly by including $C$ and $D$. It should be noted that these included terms will be exponentially small everywhere (including $y=0$ ) in the field. The solution obtained will not now satisfy

$$
\operatorname{limit}_{\zeta \rightarrow \infty} \omega_{0}(x, y, \zeta) \text { is finite }
$$


but it will satisfy the physically necessary condition

$$
\operatorname{limit}_{L \rightarrow \infty} \omega_{0}(x, y, \zeta) \text { is finite for all } x, y \text { in the field. }
$$

The above procedure adds considerable complication to the algebra and now that the feasibility of satisfying equations ( 3 ) has been demonstrated the inverse procedure will be adopted and only the negative exponentials retained.

A similar procedure to that in the previous section is applied to determine the functions $A$ and $B$. The following equation

$$
\begin{aligned}
f_{1 \zeta \zeta \zeta \zeta}\left\{u_{x}^{2}\right. & \left.+u_{y}^{2}\right\}^{2}+W_{0}^{\prime \prime}(x) u_{y}^{2} \omega_{1 \zeta \zeta} \\
& =-f_{0 \zeta \zeta \zeta \zeta}\left\{2\left(u_{x}^{2}+u_{y}^{2}\right)\left(u_{x x}+u_{y y}\right)+4\left(u_{x}^{2} u_{x x}+u_{y}^{2} u_{y y}+2 u_{x} u_{y} u_{x y}\right)\right\} \\
& -f_{0 x \zeta \zeta \zeta} 4 u_{x}\left(u_{x}^{2}+u_{y}^{2}\right)-f_{0 y \zeta \zeta \zeta} 4 u_{y}\left(u_{x}^{2}+u_{y}^{2}\right) \\
& -W_{0}^{\prime \prime}(x)\left\{2 \omega_{0 y \zeta} u_{y}+\omega_{0 \zeta} u_{y y}\right\}-W_{1}^{\prime \prime}(x) \omega_{0 \zeta \zeta} u_{y}^{2} \\
& -\omega_{0 \zeta \zeta} \omega_{0 \zeta \zeta}\left\{u_{x}^{2} u_{y y}-2 u_{x} u_{y} u_{x y}+u_{y}^{2} u_{x x}\right\}
\end{aligned}
$$

is typical of the equations from which $\omega_{1}$ and $f_{1}$ may be determined. The last term on the right hand side is quadratic in the exponentials and so does not lead to any growth in the order of $\omega_{1}$. All the other terms on the right hand side are resonant and so must vanish. This can be shown to happen provided $A$ and $B$ satisfy the differential equations

and

$$
r\left(B_{x}-A_{x}\right)+(m+n)\left(B_{y}-A_{y}\right)+p(B-A)+q B=0
$$

where

$$
r\left(B_{x}+A_{x}\right)+(m+n)\left(B_{y}+A_{y}\right)+p(B-A)-q A=0
$$

$$
\begin{aligned}
r & =8 u_{x}\left(u_{x}^{2}+u_{y}^{2}\right) \\
m & =8 u_{y}\left(u_{x}^{2}+u_{y}^{2}\right) \\
n & =2 W_{0}^{\prime \prime}(x) u_{y y} \\
p & =4\left(u_{x}^{2}+u_{y}^{2}\right)\left(u_{x x}+u_{y y}\right)+8\left(u_{x}^{2} u_{x x}+u_{y}^{2} u_{y y}+2 u_{x} u_{y} u_{x y}\right) \\
q & =-2 W_{1}^{\prime \prime}(x) u_{y y} .
\end{aligned}
$$

The characteristic lines of these pair of linear differential equations are the lines

$$
\frac{d y}{d x}=-r /(m+n)
$$

which are two-fold characteristic lines. The parabolic nature of the equations for $A$ and $B$ implies that these functions can be determined from the same boundary conditions as were used for determining the corresponding functions in the previous section. A numerical solution, based on a characteristic co-ordinate system, is possible but the arithmetic difficulties associated with 
such a solution are great because in general $u$ will only be known numerically. Thus it is apparent that the present expansion method is scarcely a practical proposition for the original physical problem from which the present simplified form was taken.

However in principle the functions $A$ and $B$ can be determined so that the terms $\omega_{0}$ and $f_{0}$ can be obtained once a suitable solution for $u(\dot{x}, y)$ is available. The solutions for $\omega_{n}$ and $f_{n}$ will follow exactly the same pattern as that for $\omega_{0}$ and $f_{0}$. Because of the introduction of the variable $\zeta$ there can never be resonant terms in the equations other than of the form

$$
\exp (-\zeta)\{\cos \zeta, \sin \zeta\}
$$

and the other difficult forms, considered in the previous section, do not occur. Thus, once the function $u(x, y)$ has been determined the asymptotic expansion can be carried out to any desired order, at least 1 in principle. There is the possibility that for a suitably chosen $p(x, y)$ the complex analysis involved may be reduced substantially and a solution useful for comparison purposes obtained. For general $p(x, y)$ however the arithmetic involved appears excessive.

\section{The function $u(x, y)$}

To complete the schematic solution process it is necessary to find a function $u(x, y)$ satisfying the differential equation (14) and the boundary. condition (13). An examination of the formal solution

where

$$
u_{v}=\frac{1}{2}\left\{K(x) \pm\left[K^{2}(x)-4 u_{x}^{2}\right]^{\frac{1}{t}}\right\}
$$

$$
K(x)=k(x) \operatorname{sign} u_{v},
$$

of equation (14) reveals the fact that there may be as many as four branches of the solution surface at any point in the field. There are no real branches if $K^{2}<4 u_{x}^{2}$, two branches if $K^{2}=4 u_{x}^{2}$ and four branches if $K^{2}>4 u_{x}^{2}$. To obtain a general solution for any of the branches it is convenient to introduce the notation

$$
\sigma=u_{x}, \quad \tau=u_{v}
$$

The characteristic form (Bernstein [10]) of the differential equation is

$$
\begin{aligned}
& \dot{x}=2 \sigma \\
& \dot{y}=2 \tau-K(x) \\
& \dot{u}=K(x) \tau \\
& \dot{\sigma}=K^{\prime}(x) \tau \\
& \dot{\tau}=\dot{0}
\end{aligned}
$$


where the dot denotes differentiation with respect to a parameter. From equations (16a, d, e) it follows that

$$
\left(\frac{\partial \sigma}{\partial x}\right)_{\tau}=\frac{1}{2} K^{\prime}(x) \tau / \sigma
$$

which integrates to

$$
\sigma=[\tau\{K(x)-T(\tau)\}]^{\frac{1}{2}}
$$

where $T$ is an arbitrary function of integration. Hence equations (16b), (16c) may be written in the form

$$
\left(\frac{\partial y}{\partial x}\right)_{\tau}=\left\{\tau-\frac{1}{2} K(x)\right\}[\tau\{K(x)-T(\tau)\}]^{-\frac{1}{2}}
$$

and

$$
\left(\frac{\partial u}{\partial x}\right)_{\tau}=\frac{1}{2} K(x) \tau[\tau\{K(x)-T(\tau)\}]^{-\frac{1}{2}}
$$

whence $y$ and $u$ may be evaluated by quadrature. The four possible branches are associated with the various combinations of sign of $\tau$ and $\sigma$.

Only one of these branches is compatible with the boundary condition (13) on $y=0$. Thus there is a unique determination of the function $u$ sufficiently close to $y=0$ but the question of other possible continuations in the large is still open. A compact form of solution for the initial branch is obtained by noting that on $y=0, u=0, \sigma=0, T=0, \tau=K(x)=k(x)$ so that, if $s$ is the value of $x$ at which a given line $\tau$ constant cuts $y=0$, the solution is given by

$$
\begin{aligned}
& y=\frac{1}{2} \int_{s}^{x}[2 k(s)-k(x)][k(s)\{k(x)-k(s)\}]^{-\frac{1}{2}} \mathrm{~d} x \\
& u=\frac{1}{2} \int_{s}^{x} k(s) k(x)[k(s)\{k(x)-k(s)\}]^{-\frac{1}{2}} \mathrm{~d} x
\end{aligned}
$$

for the case of greatest practical interest when $k^{\prime}(x)$ is positive. Similar expressions are obtainable for the other cases. The above solution indicates the existence of certain difficulties associated with this initial branch of the solution. Thus for $s$ such that $[2 k(s)-k(x)]$ is negative the integrand in the equation for $y$ is negative so that for any given value of $x$ there is a finite maximum value of $y$ for any given $k$ with positive $k^{\prime}(x)$. For certain functions $k(x)$ the initial branch of the solution will not define a solution throughout the range $0 \leqq y \leqq 1$.

In order to investigate this matter further it is convenient to consider sample solutions corresponding to particular functions $k$ rather than to discuss the general case. Suitable solutions are provided by the cases when the differential equation (14) possesses similarity solutions. Such solutions 
exist in all cases which, by suitable changes of scale, are reducible to either of the forms

$$
k(x)=\exp x \text { when } u=h(y) \exp x
$$

or

$$
k(x)=x^{m} \quad \text { when } \quad u=x^{m+1} g(\xi)
$$

where

$$
\xi=y / x .
$$

The functions $g$ and $h$ can be shown to satisfy the ordinary differential equations

$$
h^{\prime 2}+h^{2}=\left|h^{\prime}\right|
$$

and

$$
\left\{(m+1) g-\xi g^{\prime}\right\}^{2}+g^{\prime 2}=\left|g^{\prime}\right|
$$

The solution of equation (17b) with $h^{\prime}$ positive which satisfies the appropriate boundary condition is given implicitly by

$$
\arcsin 2 h-\frac{1}{2}\left\{1-\left(1-4 h^{2}\right)^{\frac{1}{2}}\right\} / h=y
$$

and this defines a suitable solution only for $0 \leqq h \leqq \frac{1}{2}$ and $0 \leqq y \leqq(\pi / 2-1)$. No solution with $h^{\prime}$ positive exists for $y$ just greater than $(\pi / 2-1)$ but it is possible to obtain a continuous function $h$ by continuing the above solution beyond the upper limit with either branch

$$
h^{\prime}=\frac{1}{2}\left\{-1 \pm\left(1-4 h^{2}\right)^{1}\right\}
$$

on which $h^{\prime}$ is negative. Either solution is acceptable on the grounds previously discussed so that the function $u$ is not determined uniquely. Moreover, corresponding to each solution $u$, there will be a distinct pair of functions $A$ and $B$ so that more than one expansion of the form (12) exists with the functions $\omega_{n}$ and $f_{n}$ having the required properties. It is to be emphasized that the method of construction guarantees that the error terms can be made small only in comparison with any power of $L^{-\frac{1}{2}}$ in the limit as $L$ tends to infinity. It does not preclude the existence of error terms, not previously considered, which are mathematically small in comparison with any term $L^{-n / 2} W_{n}(x, y)$ but not necessarily mathematically small in comparison with $L^{-n / 2} \omega_{n}(x, y, \zeta)$. Thus the alternative expansions, involving different exponential terms corresponding to different forms of $u$, can arise from rearrangements of an infinite number of terms as alternative functional forms of $x, y, \zeta, L^{-1}$. One might hope that one of these arrangements would be such that $\sum L^{-n / 2}\left(W_{n}+\omega_{n}\right)$ is convergent for sufficiently large $L$ 
and not merely asymptotic but there appears no practical manner of deciding this.

However the present aim is to obtain an expansion which will provide useful numerical results in a range of the parameter where the exponential terms which appear in the solution are not numerically negligible in comparison with powers of $L^{-\frac{1}{2}}$. As the use of limit processes must be avoided, the justification of the approximation has to lie in the examination of the residual terms in the differential equations required to make the curtailed series an exact solution. For a set of equations derived from a stable physical system it suffices to reduce the residuals to a suitable numerical order of smallness. This can be achieved in general by an expansion of the form (12) for reasonably small $L^{-\frac{1}{2}}$ because all the residuals are reduced by such a factor at each stage. With an expansion of the form (5) however not all the residuals are reduced by such a factor so that a further restriction, possibly serious, has to be placed on $L$ to ensure the smallness of the exponential terms.

With this method of justification in view it is sensible when deciding between different possible functions $u$ to choose one which enables the residuals to be reduced most rapidly. Suppose the exact function $\omega(x, y, L)$ were known and an attempt were to be made to arrange it in the form of the early terms of equation (12) in such a way as to make the residuals as small as possible. Any portion $v$ may be included as a function of $x$ and $y$ or deferred to a later term by rearranging it in the form $L^{-n / 2}\left(L^{n / 2} v\right)$.* Thus any portion of $\omega$ which is arranged in terms of $\zeta$ is deferred to a later term in the series and hence in order to reduce the residuals to as small a value as possible the average contribution of functions of $\zeta$ over the field should be made small. In order to reduce the residuals in the boundary conditions, it is necessary for the functions of $\zeta$ to take specified values on the boundary. Hence in order to achieve as efficient an expansion as possible it would appear proper to choose the branch of the solution $u$ which gives the largest value of $u$, thus making $\exp (-\zeta)$ as small as possible on the average.

If these ideas are applied to the determination of the function $h$ for $y$ greater than $(\pi / 2-1)$ it can be seen that the branch yielding the greatest value of $u$ satisfies the equation

$$
h^{\prime}=\frac{1}{2}\left\{-1+\left(1-4 h^{2}\right)^{\frac{1}{2}}\right\}
$$

for this has the lesser rate of decrease. This equation has the solution

$$
y=-2+\arcsin 2 h+\frac{1}{2}\left\{1+\left(1-4 h^{2}\right)^{-\frac{1}{2}}\right\} / h
$$

and this defines a value of $h$, positive, for all $y$ greater than $(\pi / 2-1)$.

- If included in this form it would be summed together with other such terms to give exponential functions. 
Because of the discontinuity of $u_{y}$ at the transition point between the two branches, $\omega_{0}$ possesses discontinuities of first derivatives at the point and this is not permissible for the function $\omega(x, y, L)$ for physical reasons. The source of this error can be traced by noting that

$$
h^{\prime \prime}= \pm 2 h h^{\prime}\left(1-4 h^{2}\right)^{-\frac{1}{2}}
$$

and hence $u_{y y}$ is infinite at the transition point. An examination of the expression

$$
\omega_{0 y y}=L \omega_{0 \zeta \zeta} u_{v}^{2}+L^{\frac{1}{2}} \omega_{0 \zeta} u_{y y}+2 L^{\frac{1}{2}} \omega_{0 y \zeta} u_{y}+\omega_{0 y \nu}
$$

reveals that, in the neighbourhood of the transition point, $L^{\frac{1}{2}} \omega_{0 \zeta} u_{y y} \gg$ $L \omega_{0 \zeta \zeta} u_{v}^{2}$ although, in the previous section, the later term has been used to dominate $\omega_{y y}$. Thus a boundary layer in the solution for $u$ is to be expected in which the discontinuities in the significant derivatives of $u$ are smoothed out. As this will affect $\omega_{0}$ near the point where $\omega_{0}$ is of the smallest order anywhere in the field the error in neglecting this additional term in $u$ is probably negligible in comparison with the other error terms.

The solutions of equations (18b) exhibit different properties from that of equation $(17 \mathrm{~b})$. For some values of $m$, the solution of the initial branch can be continued to infinity without difficulty. Thus for $m=-1$ the solution is $u=\arctan y / x$ while for $m=0$ it is

$$
u=y \text {. }
$$

For such solutions there is no difficulty associated with the solution method of section 4. For other values of $m$ however difficulties arise with the determination of a unique function $u$. For the case $m=1$ there are reasons associated with the original physical problem for expecting trouble and so a numerical solution of equation (18b) was obtained in this case.* It was found that the solution of the initial branch, which yields the largest value of $u$, is found to be real only for $\xi$ less than $\mathbf{0 . 3 9 3}$ and the value of $g$. there is such that none of the branches can be continued beyond this point if it is assumed that $g$ is continuous. Since a discontinuity in $g$ would involve exponentially large relative changes in $\omega_{0}$ it would appear necessary to introduce a transition region to permit a switch to be made to another branch at some $\xi$ less than the critical value 0.393 . By the use of such transitions it is desired to find a solution for the range $0 \leqq \xi \leqq \infty$ but it can be shown that the only real solution bounded at infinity is that solution of the branch with the lesser rate of decrease

$$
g^{\prime}=\left\{-1+4 \xi g+\left[1-8 \xi g-16 g^{2}\right]^{2}\right\} /\left\{2\left(1+\xi^{2}\right)\right\}
$$

* The numerical solution was obtained on SILLIAC by Mr. P. D. Jones and Mr. R. Whitfield to whom the author is indebted. 
whose asymptotic behaviour for large $\xi$ is given by

$$
g=(9 \xi)^{-1}+O\left(\xi^{-3}\right) .
$$

The graphs of this solution and the initial branch are displayed in Fig. 1. It is seen that the two solutions have a common range of definition and that at one point, namely $\xi=0.188$, they are equal. Thus a continuous solution in the entire range can be obtained by following the initial branch for $0 \leqq \xi \leqq 0.188$ and the other branch for $\xi>0.188$. It should be noted that a solution, with greater values of $u$ in portion of the range, can be obtained by following the initial branch beyond $\xi=0.188$ but not as far as $\xi=0.393$ and returning to the above solution of equation (20) via the branch,

$$
g^{\prime}=\left\{-1+4 \xi g-\left[1-8 \xi g-16 g^{2}\right]^{\frac{1}{2}}\right\} /\left\{2\left(1+\xi^{2}\right)\right\} .
$$

By switching branches more often it appears possible to obtain functions $u$ yielding even more "economical" expansions.

However this presupposes the possibility of introducing suitable transition regions in which the consequent discontinuities of derivatives of $u$ are smoothed out. Whether it is possible to switch from any one branch to any other at will is a matter for investigation of the properties of the solutions in these transition layers. This appears a formidable task. However there is one feature of the above solution which suggests that some such transition is. possible. From the behaviour of the solution for large $\xi$ it follows that

$$
\zeta=L^{\frac{1}{2}} x^{3} /(9 y)
$$

and the form of solution for $\omega_{0}$ implies the existence of a boundary layer of thickness $O\left(L^{-\frac{1}{t}}\right)$ near the origin where $x$ and $y$ are of the same order and a boundary layer of thickness $O\left(L^{-t}\right)$ along $x=0$. Mahony [6] has predicted the existence of such boundary layers on physical grounds. The fact that the present method indicates these boundary layers automatically adds to the author's confidence that the solution obtained here for $u$ is essentially correct. However it is apparent that further work is necessary to obtain a better understanding of the implications of the existence of more than one function $u$ such that the expansion (12) is satisfactorily asymptotic.

\section{Conclusions}

The original aim of the present work was to extend the method developed by Kaplun, Lagerstrom, Proudman and Pearson [1, 2, 3, 4] so that terms which were mathematically insignificant, but numerically important, could be obtained. This aim has been achieved, at least in principle for the given example, by a series of modifications of the solution obtained by the method of the cited authors. This method of presentation has been adopted 
for the insight it gives into the structure of the new method and its relationship to the previous work. However there is no reason why the gradual development, as described here, should be followed. In any further application it should prove possible, using the standard order of magnitude arguments of perturbation theory, to deduce the form of expansion corresponding to equations (12).* Thus it is not necessary to base the present method on the work of the cited authors and in fact, for reasons to be discussed below, the single expansion of the present paper may be more fundamental even when terms which are mathematically insignificant in the limit are neglected.

If exponentially small terms are negligible the single form of expansion (5) involves no extra labour as compared with the method of the cited authors. In certain cases, and the present problem is one of these, there is no real advantage to either method. However the present method appears to have a greater potential because it eliminates the matching technique which involves the expansion of certain functions in series form and then rearranging the order of terms in the series. It is improbable that these processes involved in the matching can be justified for other than special problems. However an examination of the properties of an expansion of the form (5) does provide an indication of the limitations of the matching techniques.

In order to obtain separate expansions of the form required for the matching technique it is necessary that the functions $W_{n}(x, y)$ be expansible in suitable series, not necessarily power series, for small values of $y$ and the functions $\omega_{n}(x, \eta)$ be expansible in suitable series for large $\eta$. The existence of such expansions is essential for the matching technique but not for the present method. It is doubtful whether functions will arise in physical problems which do not permit such expansions other than near isolated values of $x$. However it is easy to think of plausible non-pathological examples, such as $x^{2} /\left(x^{2}+y^{2}\right)$, which is not expansible for $y$ small near $x=0$. Problems in which singular functions arise are treated more easily by the present method as the need to expand such functions is eliminated. It is also conceivable that the rearrangement of the series involved in the matching can lead to trouble. However the author is not convinced that this is a serious difficulty for all specimen examples he has been able to construct are of an extremely artificial nature.

There may be further disadvantage deriving from the expansions which are necessary for matching. These may be illustrated by considering the case when the function $W_{2}(x, y)$ takes the form $K_{0}(y) / \ln y$ where $K_{0}$ is the Bessel function of complex argument. If this is expanded for small $y$ preparatory to matching it becomes

* Modifications of this assumed form may be necessary but should be suggested by the progress of the perturbation. 


$$
\left\{-\ln y+(\gamma+\ln 2)+O\left(y^{2}\right)\right\} / \ln y
$$

or in terms of $\eta$

$$
\left\{-1+(\gamma+\ln 2) \sum_{m=0}^{\infty}\{2 / \ln L\}^{m+1}(\ln \eta)^{m}+O(I)\right\}
$$

Such a series has an extremely poor rate of convergence and is virtually useless for numerical work. The present methorl may not enrounter such difficulties (and does not for a problem in which only the value of $W_{n}$ is involved in the boundary conditions) The above discussion has been concerned with difficulties which arise in the appliration of the matching technique but which are avoided by a cingle expansion of the form (5) How important and frequent will be such cases is an open question but the general use of an expansion of the form (5) appears advantageons esperially as it does not introduce essentially different analvsis

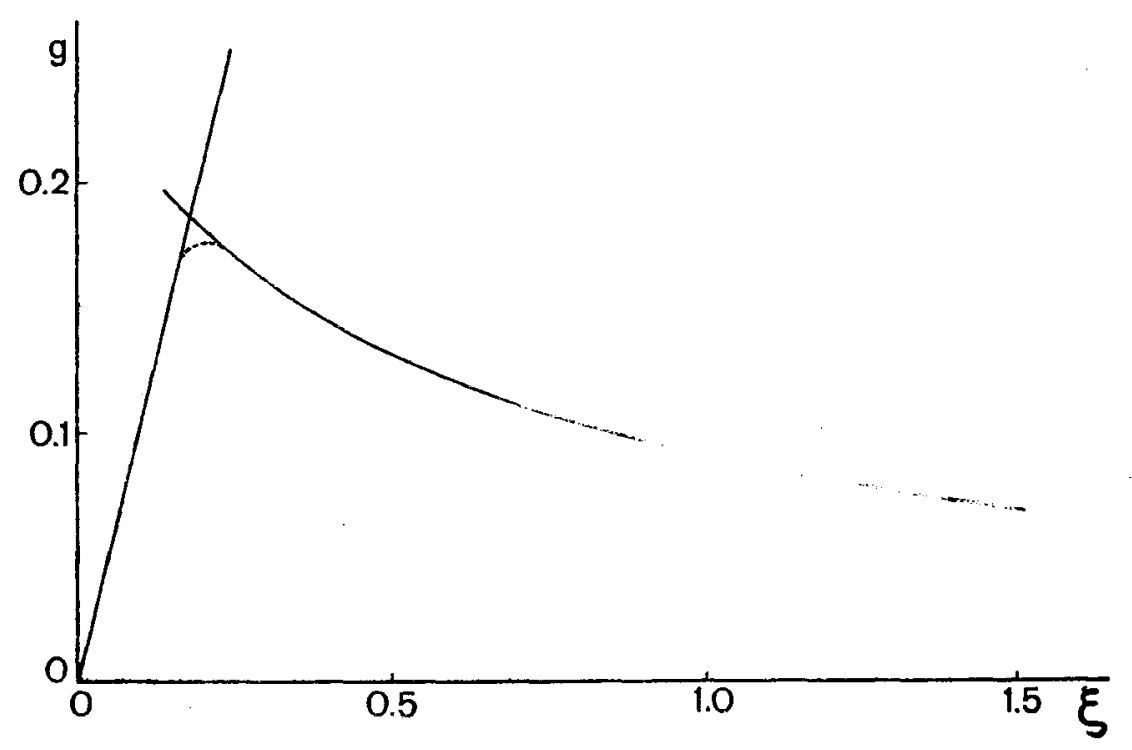

There is a further type of problem wherein a simplified form of the present method should be useful. If $L$ is sufficiently large that in general exponentially small terms are insignificant there may be small regions where this is not so. An example of this occurs in the present paper in the neighbourhood of a point where $k(x)$ vanishes. It is plausible that the effect of this will be confined to a small range of $x$ in which $k(x)$ may be approximated by a form for which the similarity solutions of the previous section may be applied. Elsewhere the exponentially small terms will be ignored. The full analysis of this paper would need to be applied only to certain suitable approximate representations. Thus such cases may be treated even when the analysis 
is impractical for the whole field. If in rettain problems in which of vanishes in the field this may provide a means of tarkling problems involving sefar at ed boundary layers.

The simplified formis of the proposed method appear to have 11seful applirations beside the use, originally envisaged, of providing terms which ate numerically significant but which are insignificant in the limit It is possible that in other puohlems the ammunt of anaiyaic involver in applving the full method will not be as prohibitive as in the present exunfle For such cases the methor conld be appliert to provide a solution of the type originally songht.

The author wishes to arknowledge several helpful suggestions he received from I) $H$ C I evey during the preparation of this paper

\section{References}

[1] Kaplun, S. and Iagerstrom, P A. J Math. and Mech. 6, (1957), 585.

[2] Kaplun, S, J. Math and Mech, 6, (1957), 595.

[3] Iagerstrom, P A., J. Math and Merh 6, (1957), 605.

[4] Froudman, I. and Pearsin, J. R. S., J. Fluid Merh. 2, (1957), 237.

[5] Mahony, J. J, Q. J. M. A. M

[6] Mahony, J. J.. Q. J. M. A. M.

[7] J.agerstrom, P. A. and Cole, J. D, J Rat. Mech and Ana1. 4, (1955), 817

[8] Lighthill, M. J., Phil. Mag. 40, (1949), 1179,

[9] Kaplun, S., Z. A. M. P. 5, (1954), 111.

[10] Bernstein, D. L., Existence Thertems in Partial J)ifferential Equatinns. 1950, Princetun Univ. Press.

Department of Mathematics

Iniversity of Queensland. 